\title{
An Enhanced Matrix-Free Secant Method via Predictor-Corrector Modified Line Search Strategies for Solving Systems of Nonlinear Equations
}

\author{
M. Y. Waziri ${ }^{1,2}$ and Z. A. Majid ${ }^{1,3}$ \\ ${ }^{1}$ Department of Mathematics, Faculty of Science, Universiti Putra Malaysia, 43400 Serdang, Selangor, Malaysia \\ ${ }^{2}$ Department of Mathematical Sciences, Faculty of Science, Bayero University Kano, Kano, Nigeria \\ ${ }^{3}$ Institute for Mathematical Research, Universiti Putra Malaysia, 43400 Serdang, Selangor, Malaysia \\ Correspondence should be addressed to M. Y. Waziri; mywaziri@gmail.com
}

Received 28 September 2012; Revised 24 December 2012; Accepted 7 January 2013

Academic Editor: Paolo Ricci

Copyright ( 2013 M. Y. Waziri and Z. A. Majid. This is an open access article distributed under the Creative Commons Attribution License, which permits unrestricted use, distribution, and reproduction in any medium, provided the original work is properly cited.

Diagonal updating scheme is among the cheapest Newton-like methods for solving system of nonlinear equations. Nevertheless, the method has some shortcomings. In this paper, we proposed an improved matrix-free secant updating scheme via line search strategies, by using the steps of backtracking in the Armijo-type line search as a step length predictor and Wolfe-Like condition as corrector. Our approach aims at improving the overall performance of diagonal secant updating scheme. Under mild assumptions, the global convergence results have been presented. Numerical experiments verify that the proposed approach is very promising.

\section{Introduction}

Consider the problem

$$
F(x)=0,
$$

with $F: R^{n} \rightarrow R^{n}$.

The mapping $F$ is assumed to satisfy the following standard assumptions:

(1) $F$ is continuously differentiable in an open convex set $\Phi$

(2) there exists a solution $x^{*}$ of (1) in $\Phi$ such that $F\left(x^{*}\right)=$ 0 and $F^{\prime}\left(x^{*}\right) \neq 0$;

(3) the Jacobian $F^{\prime}(x)$ is local Lipschitz continuous at $x^{*}$.

The famous scheme for finding the solution to (1) is the Newton method. The method is simple to implement, and it produces an iterative sequence $\left\{x_{k}\right\}$ from any given initial guess $x_{0}$ in the neighborhood of $x^{*}$ via

$$
x_{k+1}=x_{k}-\left(F^{\prime}\left(x_{k}\right)\right)^{-1} F\left(x_{k}\right),
$$

where $k=0,1,2 \ldots n$.
The attractive features of this method are that it is easy to implement and converges rapidly [1]. However, the major difficulty of Newton-type method is matrix storage requirements especially when handling large systems of nonlinear equations [1]. To overcome such difficulty, the simple modification on the Newton method is the fixed Newton method. Fixed Newton method for the determination of solution $x^{*}$ is given by

$$
x_{k+1}=x_{k}-\left(F^{\prime}\left(x_{0}\right)\right)^{-1} F\left(x_{k}\right), \quad k=0,1,2, \ldots .
$$

This method avoids computation and storing the Jacobian in each iterations (except at $k=0$ ). However, it still requires to solve systems of $n$ linear equations and consumes more CPU time as the system's dimension increases $[2,3]$.

Quasi-Newton method is another variant of Newtontype methods. It replaces the Jacobian or its inverse with an approximation which can be updated at each iteration and is given as

$$
x_{k+1}=x_{k}-B_{k}^{-1} F\left(x_{k}\right),
$$

where the matrix $B_{k}$ is the approximation of the Jacobian at $x_{k}$. The main idea behind quasi-Newton's method is to 
eliminate the evaluation cost of the Jacobian matrix $[4,5]$. However, the most critical part of such quasi-Newton method is storing the full matrix of the approximate Jacobian, which can be a very expensive task as the dimension of systems increases [6]. In contrast, this paper presents an improved variant of quasi-Newton update via the steps of backtracking in the Armijo-type line search and Wolfe-like condition and incorporating restarting strategy whenever the updating matrix is singular or nearly singular. The anticipation has been to improve the overall performance of the diagonal quasi-Newton method.

We organized the rest of this paper as follows. In the next section, we present the proposed method. Section 3 presents convergence results. Numerical experiments are reported in Section 4, and finally, conclusion is given in Section 5.

\section{The Improved Secant Diagonal Updating}

It is well known that it is not always feasible to compute the full elements of the Jacobian matrix of the given nonlinear function or it may be very expensive. We often have to approximate the Jacobian matrix by some other approach, and the famous method of doing so is quasi-Newton's method [4]. The basic idea underlining this approach has been to reduce the evaluation cost of the Jacobian matrix. This new approach generates a sequence of points $\left\{x_{k}\right\}$ via

$$
x_{k+1}=x_{k}-\alpha_{k} B_{k} F\left(x_{k}\right),
$$

where $\alpha_{k}$ is a step length, and $B_{k}$ is a diagonal approximation of the inverse Jacobian matrix which can be updated in each iterations. To achieve this, we incorporate some new line search strategies, via the steps of backtracking in the Armijo-type line search as predictor and then improved via Wolfe-like condition as a corrector. The Armijo rule is among the inexact line search methods which guarantees a sufficient degree of accuracy to ensure the algorithm convergence. Nevertheless, the scheme needs high floating points operations and function call. In this paper, we present a simple line search strategy, which is less computational cost, floating points operations and CPU time consumptions respectively compared to classical Armijor line search. The approach is as follows.

Given $\epsilon \epsilon(0,1)$ and $\sigma<1$, the proposed approach finds the appropriate $\alpha$, such that

$$
\left\|F\left(x_{k}+\alpha_{k} d_{k}\right)\right\| \leq \sigma\left\|F\left(x_{k}\right)\right\| .
$$

In addition, the new strategy is implemented in an iterative way using a fixed initial value of $\alpha$ as follows.

Algorithm 1 (Armijo-Like).

Step 0 . Set $K=0, \alpha_{0}>0$, and $\sigma<1$.

Step 1. $\left\|F\left(x_{k+1}\right)\right\| \leq \sigma\left\|F\left(x_{k}\right)\right\|$. Choose $\alpha_{k}$ as the step size; stop. Otherwise $\alpha_{k+1}=\alpha_{k} / 2, k=k+1$.

Waziri et al. [7] have set the step length $\left(\alpha_{k}=1\right.$, for all $k$ ); this approach is mostly used in many Newton-like methods.
Here, we continue in the spirit of diagonal updating, using a new line search strategy to obtain a good step length $\left(\alpha_{k}\right)$ in every iteration, anticipating to produce a more accurate approximation of the Jacobian inverse matrix and then employing restating strategy whenever the updating matrix is undefined. To this end, $B_{k+1}$ would be obtained almost similar to the diagonal updating scheme presented in [7] in which $s_{k}=-\alpha_{k} B_{k} F\left(x_{k}\right)$ instead of $s_{k}=-B_{k} F\left(x_{k}\right)$. Now, let the deviation between $B_{k+1}$ and $B_{k}$ denoted as $\Phi_{k}=B_{k+1}-B_{k}$ be minimized under some norms; the optimal solution is given as

$$
\Phi_{k}=\frac{\left(y_{k}^{T} s_{k}-y_{k}^{T} B_{k} y_{k}\right)}{\operatorname{tr}\left(\Psi_{k}^{2}\right)} \Psi_{k},
$$

where $\Psi_{k}=\operatorname{diag}\left(\left(y_{k}^{(1)}\right)^{2},\left(y_{k}^{(2)}\right)^{2}, \ldots,\left(y_{k}^{(n)}\right)^{2}\right), \sum_{i=1}^{n}\left(y_{k}^{(i)}\right)^{4}=$ $\operatorname{tr}\left(\Psi_{k}^{2}\right), y_{k}=F\left(x_{k+1}\right)-F\left(x_{k}\right), s_{k}=x_{k+1}-x_{k}$, and tr is the trace operation, respectively. The updated formula for the proposed matrix $B_{k}$ is given as [7]

$$
B_{k+1}=B_{k}+\frac{\left(y_{k}^{T} s_{k}-y_{k}^{T} B_{k} y_{k}\right)}{\operatorname{tr}\left(\Psi_{k}^{2}\right)} \Psi_{k} .
$$

To safeguard on the possibilities of generating undefined $B_{k+1}$, we let $B_{k+1}=B_{0}$, whenever this situation happens

$$
B_{k+1}= \begin{cases}B_{k}+\frac{\left(y_{k}^{T} s_{k}-y_{k}^{T} B_{k} y_{k}\right)}{\operatorname{tr}\left(\Psi_{k}^{2}\right)} \Psi_{k}, & \operatorname{tr}\left(\Psi_{k}^{2}\right) \neq 0, \\ B_{0}, & \text { otherwise. }\end{cases}
$$

Now, we can describe the algorithm for our proposed approach as follows.

Algorithm 2 (EMFM).

Step 1. Choose an initial guess $x_{0}, \sigma \in(0,1), B_{0}=I_{n}, \alpha_{0}>0$ and let $k:=0$.

Step 2. Compute $F\left(x_{k}\right)$, and if $\left\|F\left(x_{k}\right)\right\| \leq 10^{-4}$, stop.

Step 3. Compute $d=-F\left(x_{k}\right) B_{k}$.

Step 4. If $\left\|F\left(x_{k}+\alpha_{k} d_{k}\right)\right\| \leq \sigma\left\|F\left(x_{k}\right)\right\|$, retain $\alpha_{k}$ and go to 5 . Otherwise, set $\alpha_{k+1}=\alpha_{k} / 2$ and repeat 4 .

Step 5. If $\left\|F\left(x_{k}+\alpha_{k} d_{k}\right)-F\left(x_{k}\right)\right\| \geq\left\|F\left(x_{k}+\alpha_{k} d_{k}\right)\right\|-\left\|F\left(x_{k}\right)\right\|$, retain $\alpha_{k}$ and go to 6 . Otherwise, set $\alpha_{k+1}=\alpha_{k} \times 1.1$ and repeat 5.

Step 6. Let $x_{k+1}=x_{k}+\alpha_{k} d_{k}$.

Step 7. If $\left\|x_{k+1}-x_{k}\right\|_{2}+\left\|F\left(x_{k}\right)\right\|_{2} \leq 10^{-4}$, stop. Otherwise go to Step 8 .

Step 8. If $\left\|\Delta F_{k}\right\|_{2} \geq \epsilon_{1}$, where $\epsilon_{1}=10^{-4}$, compute $B_{k+1}$, and if not, $B_{k+1}=B_{0}$.

Step 9. Set $k:=k+1$ and go to 2 . 


\section{Convergence Analysis}

We present the convergence result of EMFM method by proving the existence of the step length $\alpha_{k}>0$. We will make the following assumptions on nonlinear system $F$.

Assumption 3. (i) $F$ is differentiable in an open convex set $E$ in $\mathfrak{R}^{n}$.

(ii) There exists $x^{*} \in E$ such that $\left(x^{*}\right)=0$, and $F^{\prime}(x)$ is continuous for all $x$.

(iii) $F(x)$ satisfies Lipschitz condition of order one; that is, there exists a positive constant $\mu$ such that

$$
\|F(x)-F(y)\| \leq \mu\|x-y\|,
$$

for all $x, y \in \mathfrak{R}^{n}$.

(iv) There exist constants $c_{1} \leq c_{2}$ such that $c_{1}\|\omega\|^{2} \leq$ $\omega^{T} F^{\prime}(x) \omega \leq c_{2}\|\omega\|^{2}$ for all $x \in E$ and $\omega \in \Re^{n}$.

To this end, we proceed by given the following result for the step length generated by the proposed strategies.

Theorem 4. Assume that $F$ is a strictly convex function. Suppose that the new strategies are employed with $d_{k} \neq 0$ and positive $\alpha_{k}$ exist, for all sufficiently large $k$. Then, the iterates $\left\{x_{k}\right\}$ generated by the line search algorithm have the property that

$$
\lim \left\|F\left(x_{k}+\alpha_{k} d_{k}\right)\right\|=0, \text { as } k \longrightarrow \infty .
$$

Proof. From condition (iii) of Assumption 3 and the fact that $s_{k}=x_{k+1}-x_{k}$, we have

$$
\begin{aligned}
\left\|F\left(x_{k}\right)-F\left(x_{k}+\alpha_{k} d_{k}\right)\right\|_{F} & \leq \mu\left\|s_{k}\right\|_{F} \\
& =\mu\left\|\alpha d_{k}\right\|_{F} \\
& \leq \mu|\alpha|\left\|d_{k}\right\|_{F} .
\end{aligned}
$$

Recall that

$$
\left\|F(x)-F\left(x_{k}+\alpha_{k} d_{k}\right)\right\|_{F} \leq\|F(x)\|_{F}-\left\|F\left(x_{k}+\alpha_{k} d_{k}\right)\right\|_{F} .
$$

Equation (13) gives

$$
\begin{aligned}
\left\|F\left(x_{k}\right)\right\|_{F}-\left\|F\left(x_{k}+\alpha_{k} d_{k}\right)\right\|_{F} & \leq \alpha \mu\left\|d_{k}\right\|_{F} \\
& =\mu\left\|\alpha d_{k}\right\|_{F} \\
& \leq \mu|\alpha|\left\|d_{k}\right\|_{F} .
\end{aligned}
$$

Since $\left\|d_{k}\right\| \neq 0$ and $\left\|F\left(x_{k}+\alpha_{k} d_{k}\right)\right\| \leq \sigma\left\|F\left(x_{k}\right)\right\|$, hence, it follows that

$$
\begin{gathered}
\left\|F\left(x_{k}\right)\right\|_{F}-\sigma\left\|F\left(x_{k}\right)\right\|_{F} \leq \alpha \mu\left\|d_{k}\right\|_{F}, \\
(1-\sigma)\left\|F\left(x_{k}\right)\right\|_{F} \leq \alpha \mu\left\|d_{k}\right\|_{F} .
\end{gathered}
$$

After little simplifications, we obtain

$$
\alpha \geq \frac{(1-\sigma)\left\|F\left(x_{k}\right)\right\|_{F}}{\mu\left\|d_{k}\right\|_{F}}>0 .
$$

Hence, $\alpha_{k}$ exist and are positive.
We continue to show the convergence of the iterates $\left\{x_{k}\right\}$ by recalling that

$$
\left\|F\left(x_{k}+\alpha_{k} d_{k}\right)\right\| \leq \sigma\left\|F\left(x_{k}\right)\right\| .
$$

Then, we have

$$
\begin{aligned}
\left\|F\left(x_{k}+\alpha_{k} d_{k}\right)\right\| & \leq \sigma^{2}\left\|F\left(x_{k}\right)\right\| \\
& \leq \sigma^{3}\left\|F\left(x_{k}\right)\right\|
\end{aligned}
$$

for a finite $k$, and (18) yields

$$
\left\|F\left(x_{k}+\alpha_{k} d_{k}\right)\right\| \leq \sigma^{k}\left\|F\left(x_{k}\right)\right\| .
$$

Since the algorithm terminates at $\left\|F\left(x_{k}\right)\right\|=0$, it follows from (19) that

$$
\frac{\left\|F\left(x_{k}+\alpha_{k} d_{k}\right)\right\|}{\left\|F\left(x_{k}\right)\right\|} \leq \sigma^{k} .
$$

We have

$$
\lim \frac{\left\|F\left(x_{k}+\alpha_{k} d_{k}\right)\right\|}{\left\|F\left(x_{k}\right)\right\|} \leq \lim \sigma^{k}, \quad \text { as } k \longrightarrow \infty .
$$

Therefore, due to $\sigma<1$, it implies that

$$
\lim \sigma^{k} \longrightarrow 0, \quad \text { as } k \longrightarrow \infty \text {. }
$$

hence

$$
\lim \left\|F\left(x_{k}+\alpha_{k} d_{k}\right)\right\|=0, \quad \text { as } k \longrightarrow \infty .
$$

To show the convergence results of EMFM method, we require to show that the updating matrix $B_{k}$ is bounded above and below by some positive constants. Hence, we can state the following result on the boundedness of $\left\{\left\|\Phi_{k}\right\|_{F}\right\}$ by assuming that, without loss of generality, the updating matrix (8) is always used; then, we have the following.

Theorem 5. Let $F$ satisfy Assumption 3, and let $\left\|y_{k}\right\| \neq 0$ for all finite $k$. Let $\left\{B_{k}\right\}$ be the sequence generated by (8). If the given nonsingular $B_{0}$ satisfies

$$
\theta \leq B_{0}^{i} \leq \omega, \quad i=1,2, \ldots, n,
$$

for some constants $\theta$ and $\omega$, then the sequence $\left\{\left\|B_{k}\right\|_{F}\right\}$ is bounded for all finite $k$.

Proof. Since $B_{k+1}=B_{k}+\Phi_{k}$, it follows that

$$
\left\|B_{k+1}\right\|_{F} \leq\left\|B_{k}\right\|_{F}+\left\|\Phi_{k}\right\|_{F} .
$$

For $k=0$ and assuming $B_{0}=I$, we have

$$
\begin{aligned}
\left|\Phi_{0}^{(i)}\right| & =\left|\frac{y_{0}^{T} s_{0}-y_{0}^{T} B_{0} y_{0}}{\operatorname{tr}\left(\Psi_{0}^{2}\right)}\left(y_{0}^{(i)}\right)^{2}\right| \\
& \leq \frac{\left|y_{0}^{T} s_{0}-y_{0}^{T} B_{0} y_{0}\right|}{\operatorname{tr}\left(\Psi_{0}^{2}\right)}\left(y_{0}^{(\max )}\right)^{2},
\end{aligned}
$$


where $\left(y_{0}^{(\max )}\right)^{2}$ is the largest element among $\left(y_{0}^{(i)}\right)^{2}, i=$ $1,2, \ldots, n$.

After multiplying $(26)$ by $\left(y_{0}^{(\max )}\right)^{2} /\left(y_{0}^{(\max )}\right)^{2}$ and substituting $\operatorname{tr}\left(\Psi_{0}^{2}\right)=\sum_{i=1}^{n}\left(y_{0}^{(i)}\right)^{4}$, we have

$$
\left|\Phi_{0}^{(i)}\right| \leq \frac{\left|y_{0}^{T} s_{0}-y_{0}^{T} B_{0} y_{0}\right|}{\left(y_{0}^{(\max )}\right)^{2} \sum_{i=1}^{n}\left(y_{0}^{(i)}\right)^{4}}\left(y_{0}^{(\max )}\right)^{4} .
$$

Since $\left(y_{0}^{(\max )}\right)^{4} / \sum_{i=1}^{n}\left(y_{0}^{(i)}\right)^{4} \leq 1$, then (27) turns into

$$
\left|\Phi_{0}^{(i)}\right| \leq \frac{\left|y_{0}^{T} F^{\prime}(x) y_{0}-y_{0}^{T} B_{0} y_{0}\right|}{\left(y_{0}^{(\max )}\right)^{2}} .
$$

From Assumption 3 and $B_{0}=I$, (28) becomes

$$
\left|\Phi_{0}^{(i)}\right| \leq \frac{|c-1|\left(y_{0}^{T} y_{0}\right)}{\left(y_{0}^{(\max )}\right)^{2}}
$$

where $c=\max \left\{\left|c_{1}\right|,\left|c_{2}\right|\right\}$.

Since $\left(y_{0}^{(i)}\right)^{2} \leq\left(y_{0}^{(\max )}\right)^{2}$ for $i=1, \ldots, n$, it follows that

$$
\left|\Phi_{0}^{(i)}\right| \leq \frac{n|c-1|\left(y_{0}^{(\max )}\right)^{2}}{\left(y_{0}^{(\max )}\right)^{2}} .
$$

Hence, we obtain

$$
\left\|\Phi_{0}\right\|_{F} \leq n^{3 / 2}|c-1| .
$$

Suppose that $\eta=n^{3 / 2}|c-1|$; then,

$$
\left\|\Phi_{0}\right\|_{F} \leq \eta
$$

From the fact that $\left\|B_{0}\right\|_{F}=\sqrt{n}$, it follows that

$$
\left\|B_{1}\right\|_{F} \leq \beta,
$$

where $\beta=\sqrt{n}+\eta>0$.

\section{Numerical Results}

In this section, we consider some benchmark problems to illustrate the performance of the method proposed in this paper for solving large-scale systems of nonlinear equations when compared to some Newton-like methods. The computations are performed in MATLAB 7.0 using double precision computer, and the stopping rule used is

$$
\left\|s_{k}\right\|+\left\|F\left(x_{k}\right)\right\| \leq 10^{-4} \text {. }
$$

The identity matrix has been chosen as an initial approximate Jacobian inverse. We further design the codes to terminate whenever one of the following happens:

(i) the number of iteration is at least 250 , but no point of $x_{k}$ that satisfies (34) is obtained;

(ii) CPU time in seconds reaches 250;

(iii) There is insufficient memory to initiate the run.
The performances of these methods are be compared in terms of number of iterations and CPU time in seconds. In the following, some details on the benchmarks test problems are presented.

Problem 1. System of $n$ nonlinear equations is as follows:

$$
\begin{aligned}
f_{i}(x) & =x_{i}-3 x_{i}\left(\frac{\sin x_{i}}{3}-0.66\right)+2 \\
i & =1,2, \ldots, n, x_{0}=(3,3, \ldots, 3) .
\end{aligned}
$$

Problem 2. Extended Trigonometric function of Spedicator [8] is as follows:

$$
\begin{aligned}
f_{i}(x)=n-\sum_{j=1}^{n} \cos x_{j}+i\left(1-\cos x_{i}\right) \\
-\sin x_{i}+\exp ^{\left(1-\cos x_{i}\right)}-\sum_{j=1}^{n}\left(x_{j}+1\right), \\
i=1, \ldots, n, x_{0}=\left(\frac{1}{n}, \frac{1}{n}, \ldots, \frac{1}{n}\right) .
\end{aligned}
$$

Problem 3. System of $n$ nonlinear equations is as follows:

$$
\begin{array}{r}
f_{i}(x)=x_{i}-0.1 x_{i+1}^{2}, \quad f_{n}(x)=x_{n}-0.1 x_{1}^{2}, \\
i=1, \ldots, n-1, x_{0}=(7,7, \ldots, 7) .
\end{array}
$$

(A1) Nonadiabatic Stirred Tank Reactors. The first application is the model of [9]. The model deals with two continuous nonadiabatic stirred tank reactors. The reactors are in a series, at steady state having a recycle component, and with an exothermic first-order irreversible reaction. By eliminating certain variables, the model results into two nonlinear systems of equations as follows:

$$
\begin{aligned}
& f_{1}=(1-\lambda)\left[\frac{D}{10\left(1+\beta_{1}\right)}-x_{1}\right] \exp \left(\frac{10 x_{1}}{1+\left(10 x_{1} / \gamma\right)}\right)-x_{1}, \\
& f_{2}= x_{1}-\left(1+\beta_{2}\right) x_{2}+(1-\lambda) \\
& \quad \times\left[\frac{D}{10}-\beta_{1} x_{1}-\left(1+\beta_{2} x_{2}\right)\right] \exp \left(\frac{10 x_{2}}{1+\left(10 x_{2} / \gamma\right)}\right) .
\end{aligned}
$$

The dimensionless temperatures of the two reactors are represented by $x_{1}$ and $x_{2}$. The parameters $\lambda, \gamma, \beta_{1}, \beta_{2}$, and $D$ are given as $1,1000,2,2$, and 22, respectively.

(A2) Navigation by Range Measurements. Consider two beacons determining position by measuring the distances $r 1$ and $r 2$, where $(p 1, p 2)$ is the position of beacon $1,(q 1, q 2)$ is the position of beacon 2 , and $(u, v)$ is an unknown point (see Figure 1). This can be modeled into a two nonlinear systems of equations as follows:

$$
\begin{aligned}
& f_{1}=\sqrt{\left(p_{1}-u\right)^{2}+\left(q_{1}-v\right)^{2}}-r_{1}=0, \\
& f_{1}=\sqrt{\left(p_{2}-u\right)^{2}+\left(q_{2}-v\right)^{2}}-r_{2}=0 .
\end{aligned}
$$


TABLE 1: Numerical comparison of NM, FN, BM, I-VDN, and EMFM methods.

\begin{tabular}{|c|c|c|c|c|c|c|c|c|c|c|c|}
\hline \multirow{2}{*}{ Prob } & \multirow{2}{*}{ Dim } & \multicolumn{2}{|c|}{$\mathrm{NM}$} & \multicolumn{2}{|c|}{ FN } & \multicolumn{2}{|c|}{$\mathrm{BM}$} & \multicolumn{2}{|c|}{ I-VDN } & \multicolumn{2}{|c|}{ EMFM } \\
\hline & & NI & $\mathrm{CPU}$ & NI & CPU & NI & CPU & NI & $\mathrm{CPU}$ & NI & $\mathrm{CPU}$ \\
\hline 1 & 25 & 4 & 0.031 & 11 & 0.016 & 8 & 0.015 & 8 & 0.006 & 6 & 0.001 \\
\hline 2 & 25 & 4 & 0.062 & 11 & 0.031 & 8 & 0.016 & 8 & 0.002 & 7 & 0.001 \\
\hline 3 & 25 & 6 & 0.031 & - & - & 10 & 0.031 & 10 & 0.001 & 7 & 0.001 \\
\hline 1 & 50 & 4 & 0.046 & 11 & 0.031 & 8 & 0.046 & 8 & 0.011 & 6 & 0.008 \\
\hline 2 & 50 & 4 & 0.109 & 11 & 0.062 & 8 & 0.034 & 8 & 0.010 & 7 & 0.005 \\
\hline 3 & 50 & 6 & 0.156 & - & - & 10 & 0.124 & 10 & 0.008 & 7 & 0.004 \\
\hline 1 & 100 & 4 & 0.064 & 11 & 0.032 & 8 & 0.048 & 8 & 0.014 & 6 & 0.010 \\
\hline 2 & 100 & 4 & 0.125 & 11 & 0.046 & 8 & 0.032 & 8 & 0.015 & 7 & 0.011 \\
\hline 3 & 100 & 6 & 0.508 & - & - & 10 & 0.187 & 10 & 0.031 & 7 & 0.015 \\
\hline 1 & 1000 & 4 & 1.919 & 12 & 0.7332 & 9 & 2.122 & 9 & 0.033 & 6 & 0.018 \\
\hline 2 & 1000 & 4 & 2.277 & 12 & 0.998 & 8 & 1.778 & 8 & 0.049 & 5 & 0.031 \\
\hline 3 & 1000 & 6 & 88.031 & - & - & 10 & 13.057 & 10 & 0.041 & 7 & 0.024 \\
\hline
\end{tabular}

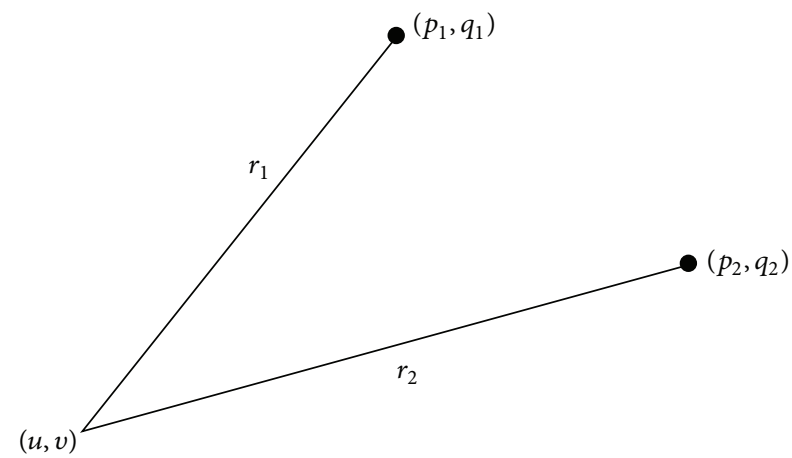

FIgURE 1

The parameters $\left(p_{1}, q_{1}\right),\left(p_{2}, q_{2}\right), r_{1}$, and $r_{2}$ are given as $(10,10),(10,-10), 14$, and 16 , respectively.

Table 1 shows the number of iterations and CPU time for these five methods, respectively. In Table 1, the value "Dim" denotes the dimension of the systems, "NI" denotes number of iterations, while "CPU" is the CPU time in seconds respectively. We analyze the performance of each method via execution time, floating points operations, and storage locations. One can observe that EMFM has the smallest number of iterations compared to the classical diagonal updating (I-VDN) proposed by Waziri et al. [7]. This shows that the line search strategies presented in this paper have increased the convergence speed of the classical diagonal updating method.

If we compare the performance of all methods, in terms of CPU time, it is clear that EMFM method consumes less CPU time than the others and still keeping memory requirement and CPU time in seconds to only $O(n)$. All five methods are able to obtain the solution $\left(x^{*}=-1.1243,1.5001\right)$ of A2, but EMFM method consumes less CPU time in second (0.001) compared to the other 4 methods. Moreover, for A1, still proposed method has shown a promising performance with less storage locations (2 locations) whereas NM, FN, and BM, respectively, required 4 locations for each.

\section{Conclusion}

From the fact that there is a rapid development in research on solving nonlinear systems, nevertheless, the dimension of the nonlinear system is most of the times so large that it requires a very costly arithmetic operations when using some other Newton-like methods; so we usually employ cheap iterative approach, and the good candidate is classical diagonal updating. Notwithstanding, the classical updating scheme has some lapses. One is that it usually needs high number of iterations. The other is that the method generally has slow convergence behavior. This paper proposes an enhanced diagonal secant updating scheme based on the steps of backtracking in the Armijo-type line search and then improved via Wolfe-like condition. Our approach aims at improving the overall performance of diagonal secant updating scheme. The algorithm only requires to store a row vector while ignoring all the off and low diagonal elements and therefore largely reduces memory locations. In addition, as it uses two line search strategies (predictor and corrector) to obtain a new iterates point, the spectral properties of the diagonal updating scheme is improved, and rapid convergence property is gained.

Computational experiment suggests that it is very vital for diagonal updating scheme to use line search strategy. EMFM method has very good solving speed and the best performance among the Newton-like methods. Finally, it can be concluded that this approach would certainly be quite useful for solving large-scale systems of nonlinear equations.

\section{References}

[1] J. E. Dennis, Jr. and R. B. Schnabel, Numerical Methods for Unconstrained Optimization and Nonlinear Equations, Prentice Hall, Englewood Cliffs, NJ, USA, 1983.

[2] M. Y. Waziri, W. J. Leong, M. A. Hassan, and M. Monsi, "A New Newton method with diagonal Jacobian approximation for 
systems of Non-Linear equations," Journal of Mathematics and Statistics, vol. 6, no. 3, pp. 246-252, 2010.

[3] M. Y. Waziri, W. J. Leong, and M. Mamat, "A two-step matrixfree secant method for solving large-scale systems of nonlinear equations," Journal of Applied Mathematics, vol. 2012, Article ID 348654, 9 pages, 2012.

[4] C. G. Broyden, "A class of methods for solving nonlinear simultaneous equations," Mathematics of Computation, vol. 19, pp. 577-593, 1965.

[5] K. Natasa and L. Zorna, "Newton-like method with modification of the right-hand vector," Mathematics of Computation, vol. 71, pp. 237-250, 2001.

[6] C. T. Kelley, Iterative Methods for Linear and Nonlinear Equations, vol. 16, SIAM, Philadelphia, Pa, USA, 1995.

[7] M. Y. Waziri, W. J. Leong, and M. A. Hassan, "Diagonal Broyden-like method for large-scale systems of nonlinear equations," Malaysian Journal of Mathematical Sciences, vol. 6, no. 1, pp. 59-73, 2012.

[8] E. Spedicator, "Computational experience with quas-Newton algorithms for minimization problems of moderately large size," Tech. Rep. CISE-N-175, Segrate, Milano, Italy, 1975.

[9] J. Sinkule, "Multiplicity and stability in a sequence of two nonadiabatic non-isothermal CSTR," Chemical Engineering Sceinces, vol. 35, pp. 987-996, 1980. 


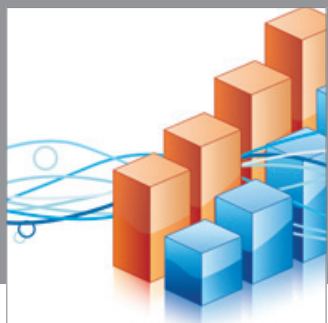

Advances in

Operations Research

mansans

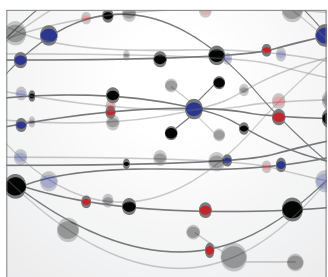

The Scientific World Journal
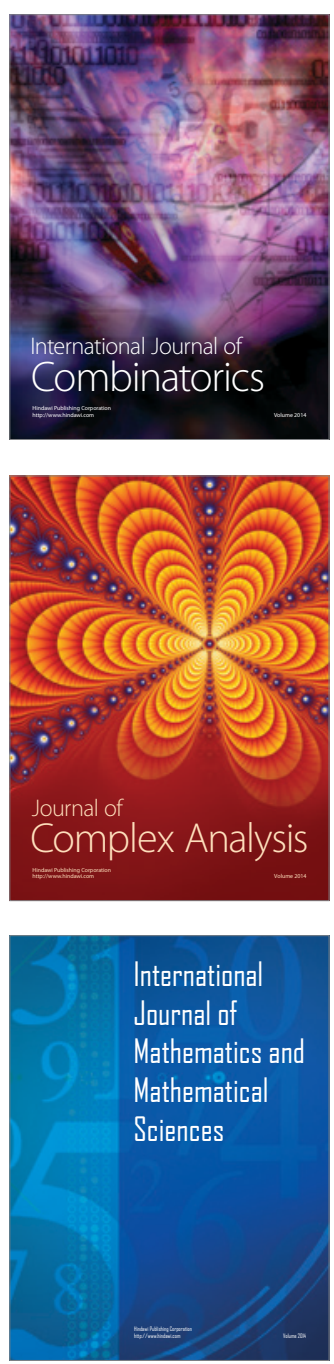
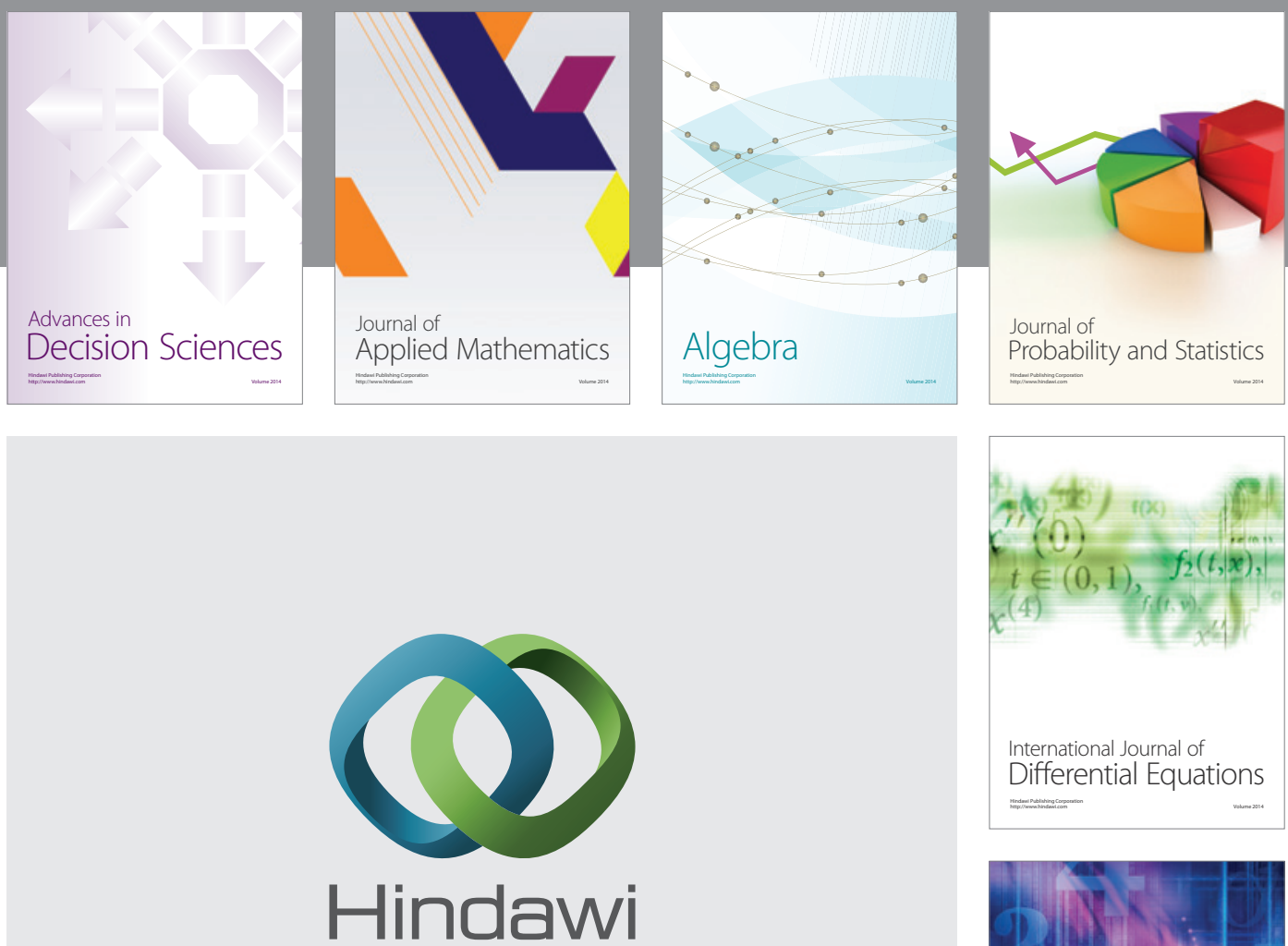

Submit your manuscripts at http://www.hindawi.com
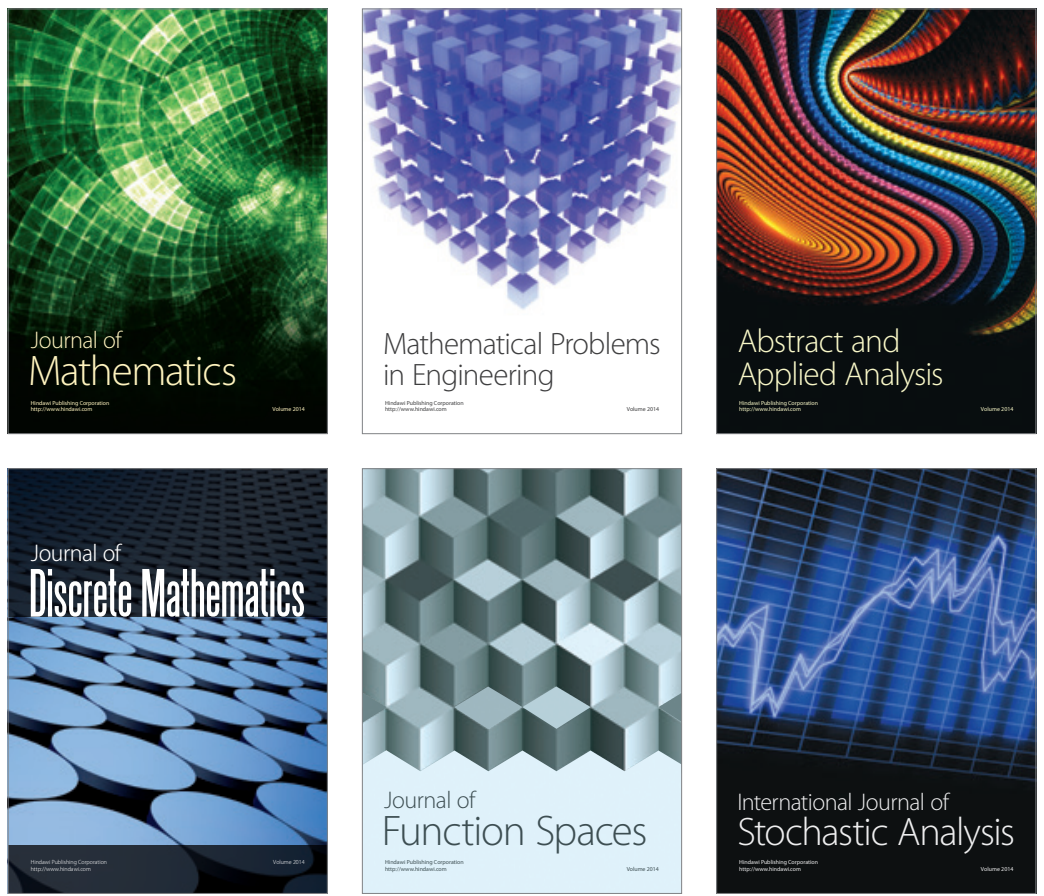

Journal of

Function Spaces

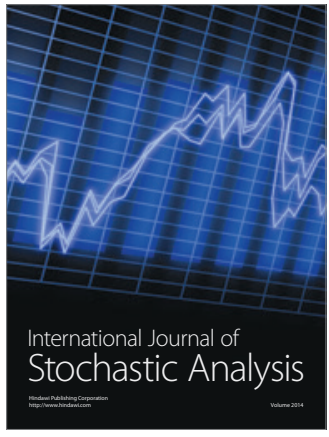

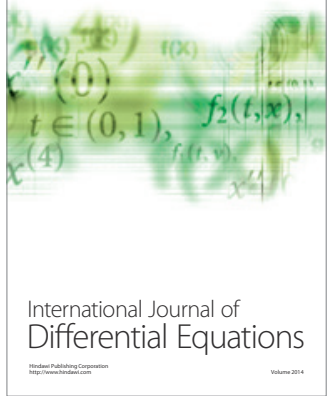
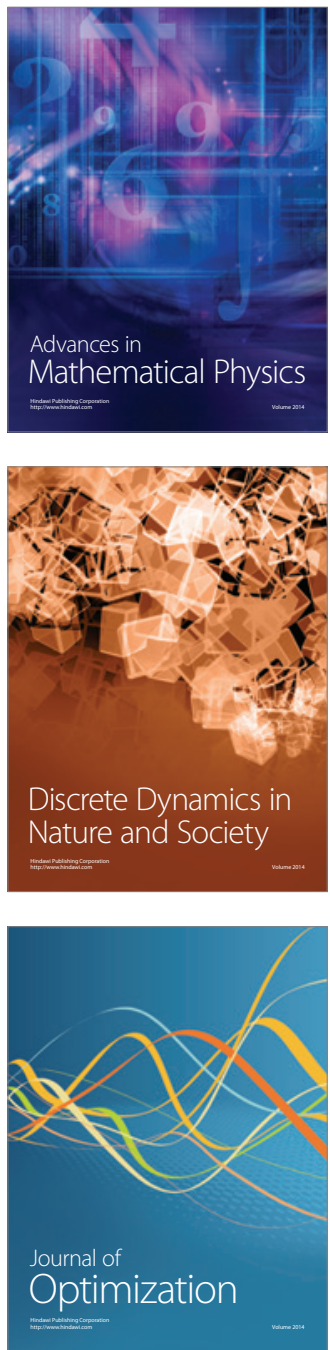